\title{
Handedness in schizophrenia and schizoaffective disorder in an afrikaner founder population
}

\author{
RH Mataboge ${ }^{1 *}$, M Joubert ${ }^{1}$, JC Jordaan², F Reyneke ${ }^{2}$, JL Roos ${ }^{1}$ \\ ${ }^{1}$ University of Pretoria, Department of Psychiatry, Pretoria, South Africa \\ ${ }^{2}$ University of Pretoria, Department of Statistics, Pretoria, South Africa
}

\begin{abstract}
Objective: An association between the Leucine-rich repeat trans membrane neuronal 1 gene (LRRTMl), schizophrenia/ schizoaffective disorder and handedness was recently claimed to be established. This study aimed to test the hypothesis that Afrikaner patients with schizophrenia/schizoaffective disorder are more non-right handed than their non-affected firstdegree relatives and that of two separate control groups. The association between handedness, gender and age at onset of illness in the patients group was also determined. Method: Two cross-sectional studies were carried out, which compared the handedness of a group of 100 (30 females and 70 males) Afrikaner patients with schizophrenia/schizoaffective disorder, their non-affected first-degree relatives, and two separate control groups. Handedness was determined by the Edinburg Handedness Inventory (EHI). Results: Patients were found to be more right-handed than expected with only 17 out of 100 being non-right-handed compared to 11 out of 100 non-affected relatives; 36 out of 100 students and 75 out of 500 nonaffected Afrikaner participants. The students were significantly more non-right handed than the patient and family groups but no difference in handedness was found when comparing the patients, family members and 500 participant control group. There was no significant difference between age at onset of illness and handedness. A significant association between female gender and non-right handedness in the patient group was found but no gender difference in the family and student groups. A highly significant association was found between the male gender of the patients and student control group regarding handedness. A significant difference was found between the median EHI raw scores of the patients and the family members. The median EHI raw scores of the patients were significantly lower than their paired family members. Conclusion: The study failed to prove a leftward shift of handedness in the patients with Schizophrenia and Schizoaffective disorder in the Afrikaner Founder population. Whether or not LRRTMl is indeed the genetic basis of handedness, remains unclear.
\end{abstract}

Keywords: Handedness; LRRTM1; Schizophrenia; South Africa

Received date: 11-11-2013

Accepted date: $25-12-2013$

doi: http://dx.doi.org/10.4172/1994-8220.1000102

\section{Introduction}

"Is LRRTMl the gene for handedness?" This question was the title of McManus, Nicholas and Vallortigara's editorial in the journal Laterality. . If the answer to this question is "yes", a natural question that follows is: what is the association between the LRRTMl gene, handedness and schizophrenia/ schizoaffective disorder? The hypothesis that schizophrenia arises from an abnormality in cerebral lateralization has gained support in recent years from studies showing anomalies in the direction and strength of lateral bias (e.g. hand, eye and foot preferences). However, results of studies investigating this topic are inconsistent. Many explanations for the anomalous patterns of lateralization have been proposed, although none have been validated.

A study by Franck et al. claimed that the first potential gene influencing human handedness - namely, the LRRTMl (Leucine-rich repeat transmembrane neuronal 1) gene on chromosome 2 - was identified in a group of siblings with dyslexia 2 . In the same study the haplotype that implicated the gene LRRTMl showed to be over-transmitted paternally

Correspondence

Dr RH Mataboge

Department of Psychiatry, University of Pretoria,

Weskoppies Hospital, P/Bag X113, Pretoria, 0001, South Africa,

email: blanch_mat@yahoo.com to patients with schizophrenia/schizoaffective disorder. This finding indicates a potential association between the LRRTMl gene, schizophrenia/schizoaffective disorder, and handedness. A genetic study conducted on 1002 affected families of European descent - including an Afrikaner sample that was recruited from the Weskoppies catchment area - has presented evidence for an involvement of the LRRTMl gene in handedness and schizophrenia. However, the relationship between the LRRTMl gene, handedness, and schizophrenia has not yet been clinically tested in the Afrikaner Founder population. ${ }^{2}$

In a study by Kenneth et al. it was argued that if schizophrenia results from a genetically determined failure of cerebral lateralization, then non-right handedness should be in excess among relatives of patients with schizophrenia. ${ }^{3}$ Kenneth et al.'s study found that relatives of the patients were more likely to be mixed handed than the controls, which tends to support the genetic etiological model. ${ }^{3}$ Further, in a study by Delisi et al. it was hypothesized that if atypical handedness is associated with the inheritance of schizophrenia, handedness will not only be shown to be familial but also to be inherited along with schizophrenia within families. ${ }^{4}$ If the hypothesis holds true, non-right handedness should be more frequently found in individuals who have schizophrenia and their mentally ill relatives compared with their mentally well relatives. 
A study by Dollfus et al. argued that one of the reasons for the inconsistent findings of handedness in patients with chronic schizophrenia is familial sinistrality i.e. an excess of mixed handedness. Their study investigated the strength of manual lateralization of patients with first episode psychosis taking into account familial sinistrality. ${ }^{5}$ Their results supported reduced manual preference i.e. mixed handedness only for patients without familial lateralization and therefore concluded that reduced manual lateralization appears to be due to the illness rather than the influence of left-handed relatives.

In the current literature the majority of studies investigating the handedness of patients with schizophrenia showed a leftward shift in the handedness distribution compared to the controls ${ }^{6-9}$. Even though it has been claimed that the leftward shift is due to predominant mixed handedness, the review by Dragovic et al. shows that the shift is due to both mixed- and left handedness ${ }^{6}$.

Schizophrenia is a heterogeneous neuropsychiatric disorder that affects roughly $1 \%$ of the adult human population. It is equally prevalent in men and women, although onset of illness in schizophrenia occurs earlier in men than women. The age at onset of the illness is also used as one of the prognostic predictors: the earlier the onset of illness the worse the prognosis of the schizophrenia. The relationship between gender, atypical handedness and schizophrenia has also been studied but the results are inconsistent. Some studies have found no gender differences in relation to handedness in schizophrenia whereas several authors have found an association between non-right handedness and the male gender ${ }^{10-15}$. In contrast, a study by Kenneth et al. showed that patients with schizophrenia and their mentally well relatives had an excess of mixed handedness compared to controls, and this excess was specific to female patients suffering from schizophrenia. ${ }^{3}$ This finding is further supported by a study by Delisi et al. in which it was found that in all indices of handedness and hand skill, females were more lateralized than males regardless of diagnosis. ${ }^{4}$

To date, few studies have investigated lateral bias in early-onset schizophrenia. Lishman and Mc-Meekan reported a shift towards non-right-handedness in young patients relative to population norms and controls. ${ }^{16}$ Oddy and Lobstein found that ambidextrous patients with schizophrenia were significantly younger than right-handed patients. ${ }^{17}$ This anomaly in hand preference suggests that lateralization is deviant in early-onset schizophrenia. The degree to which this deviation differs from the onset of schizophrenia amongst the older schizophrenia population is not yet clear, but the evidence suggests that anomalous hand preference may be more common or possibly more severe in patients with early-onset schizophrenia. ${ }^{18}$

Although the etiology of handedness, brain asymmetry and schizophrenia is likely to be complex, the discovery of the LRRTMl gene may reveal new insights in establishment/ maintenance of normal/abnormal brain development, and its relationship with diseases such as schizophrenia. ${ }^{19}$ By clinically assessing the possible relationship between handedness, schizophrenia and the LRRTMl gene, further light may be shed on the importance of the LRRTMl gene in schizophrenia, which may pave the way for further research.

The aims of this study were to test the hypothesis that patients from an Afrikaner Founder population who are suffering from schizophrenia and/or schizoaffective disorder (some of whom participated in the LRRTMl study) are more non-right handed (mixed handed or left handed) than their non-affected first-degree relatives and that of two healthy control groups. The study further aimed to determine if an association exists between handedness, gender and age at onset of illness in the same group of patients.

\section{Method \\ Study design}

Two cross-sectional studies involving two separate Afrikaner control groups were performed. Handedness of Afrikaner patients suffering from schizophrenia/ schizoaffective disorder was compared with that of their non-affected first-degree relatives and two separate control groups. The first control group consisted of Afrikaner medical students studying at the University of Pretoria (UP), South Africa and the second control group consisted of 500 Afrikaner participants from the general population who were recruited at various schools, churches and old age homes in Pretoria, South Africa.

The reason for the two separate control groups was due to the unexpected finding of the significant non-right handedness of the original student control group; which was a serious limitation to interpreting the study results. In order to address this limitation, a second larger control group consisting of Afrikaner participants from various educational and economic backgrounds was recruited.

\section{Participants and eligibility criteria}

Patients suffering from schizophrenia or schizoaffective disorder were recruited from an ongoing collaborative project between the Laboratory of Human Neurogenetics at the Columbia University in New York, USA, and the Department of Psychiatry at the University of Pretoria, South Africa. This genetic study was conducted on the genetically isolated Afrikaner founder population of South Africa. The criteria used for being an Afrikaner were: Afrikaans as a first language; having typical Afrikaans surnames of both parents and grandparents on paternal and maternal side; and genealogical tracings by a genealogist.

The diagnostic instrument of choice in the original genetic study was the 'Diagnostic Interview for Genetic Studies DIGS'20 which was translated into Afrikaans. A more detailed description of the sample, including sample collection, clinical evaluation and genealogical research can be found in a study by Karayiorgou et al. ${ }^{21}$.

A convenience sample of 100 patients from Weskoppies Hospital, Pretoria, and catchment areas was recruited from the abovementioned existing cohort of a previous genetic study on schizophrenia. Each patient was paired with a firstdegree relative who was not affected by schizophrenia/ schizoaffective disorder or any other psychotic disorder. If the particular first-degree relative could be contacted and interviewed, the specific patient-first-degree-relative pair was included in the sample. The investigators then contacted the patients and their first-degree relatives either telephonically or in person for voluntary participation. The patient-first-degree-relative pairs were not statistically randomized but the selection was blind to handedness and the investigators included the first 100 pairs who were willing to participate. Although the family group consisted of non-affected first-degree relatives, there existed a correlation between the patients and family members. 
In the statistical analyses where the family group and the patients were compared, paired groups were used instead of independent groups. Some of the patients included had also participated in the LRRTMl study by Francks et al. ${ }^{19}$. The age at onset of illness was extrapolated from the DIGS Summary Reports. This age at onset of illness was defined as: the emergence of the first psychotic symptoms, according to subjective reports and information obtained from the DIGS Summary Reports.

For the control group, a stratified random sample of 200 Afrikaner medical students enrolled at the University of Pretoria in 2009 was selected by the statistician. The criteria used for being Afrikaner were the same as for the patient group, except for the genealogical tracings. The first 100 students willing to participate and without a history or family history of a psychotic disorder were included in the study. The students (61 males and 39 females) were approached at the School of Medicine at the University of Pretoria and were requested to complete a questionnaire. The students who participated in the study were fairly equally represented across the years of study from the second year to the sixth. The selection of the students was blind to handedness.

It can be argued that the medical students are a very selected and bias group of well educated, economically advanced participants and with probably much greater experience of filling in questionnaires. In order to address this selection bias of medical students, a more appropriate and larger second control group was recruited at various prior selected old age homes, churches and schools. This second control group consisted of 500 Afrikaner participants from the general population with various economic and educational backgrounds. The first 500 Afrikaner members of the general population who were willing to participate and without a history or family history of a psychotic disorder were included in the study.

\section{Measure of handedness}

The handedness of all participants was tested by means of the Edinburgh Handedness Inventory (EHI), which is a scale used to assess the dominance of a person's right- or left hand in everyday activities. Each participant was asked to describe how he/she would perform each of ten activities. The ten activities used for testing handedness were writing, drawing, throwing, using scissors, using a toothbrush, using a knife (without a fork), using a spoon, using a broom (upper hand), striking match and opening box (lid). Some of the interviews were carried out telephonically. Handedness was scored numerically. The preference in the use of hands was indicated by noting "+" in the appropriate column (left or right). Where the preference was so strong that the participant would never use the other hand, " ++ " was noted. If there was no preference, a "+" was noted in both columns. The ten items were used to calculate a laterality quotient (LQ) in adding all the "+s" for each hand, subtracting the sum of the left from the right, and dividing the difference by the sum of both and multiplying the answer by 100 . The LQ ranges from -100 (totally left handed) to +100 (totally right handed). Participants with a score of -100 to -60 were classified as left handed and the participants with a score of +60 to +100 as right handed; those with scores -59 to +59 were classified as mixed handed. The cut-off points that best separated mixed handedness from single left- and right handedness were studied by Dragovic and Hammond and were statistically found to be between $0+/-50$ to $0+/-$ 70 , with the best statistical agreement at the $0+/-60$ cut-off.
6,22 The EHI is a reliable and validated instrument and most widely used in research. The raw EHI scores as well as the 0 $+/-60$ cut-off points were used in the analysis.

\section{Statistical Analysis Sampling design}

The sample of students was stratified to reflect the gender composition of the patients diagnosed with schizophrenia/ schizoaffective disorder, so that roughly $70 \%$ of the student sample were male and $30 \%$ female. Convenience sampling was used for both the patients as well as the other control group. The second control group was a convenience sample.

\section{Data management and statistical methods}

Information on the demographic characteristics of the participants was gathered by means of a structured questionnaire and the handedness of the subject was determined by administering the EHI. The result of the EHI test was also recorded on the questionnaire. The information in the questionnaires was captured in Excel and the data was then imported into the statistical package SPSS. All statistical analysis was performed in SPSS version 20.0, released in 2012. There were dependent and independent groups. Therefore different statistical techniques were used. Pearson's chi square test was performed to establish any association between handedness and the presence of schizophrenia/schizoaffective disorder in the Afrikaner Founder population. However, the chi square test of independence is based on the assumption that the expected frequency in each cell of the contingency table is at least 5 to ensure that the chi-squared distribution provides an adequate approximation of the sampling distribution. In cases where this assumption was not met, Fisher's Exact test was performed. The McNemar test was used to test for an association between dependent groups (patients and family members). The t-test for independent groups was used to test for the difference of means. The Kruskall-Wallis test was performed to test for the difference of medians between more than two groups. The Mann Whitney U test was used to do pair wise comparisons of the median raw scores across the patient group versus the two control groups as well as between the two control groups. The Wilcoxon Signed ranks test was used to test for the difference in the median EHI scores of the patients and the family members. The distribution of raw EHI scores for all groups was skew to the left, therefore the one way ANOVA could not be used.

\section{Ethical considerations and consent}

Approval for the study was obtained from the Research Ethics Committee of the Faculty of Health Sciences of the University of Pretoria. Written or verbal consent (in the case of telephonic interviews) was obtained from all participants. Permission to involve the medical students in this study was obtained from the Dean of the School of Medicine at the University of Pretoria. To ensure confidentiality and anonymity, each participant in the study was assigned a unique study number. Patients' identities were known only to the researchers.

\section{Results}

\section{Gender distribution within groups}

The patient group consisted of 100 participants of whom 70 were male and 30 female. Eighty (80) of the 100 patients were diagnosed with schizophrenia, while 20 were diagnosed with schizoaffective disorder: 16 with a bipolar 
subtype and 4 with a depressive subtype of schizoaffective disorder. In contrast to the patient group where the gender composition was 70 males and 30 females, the familymember group consisted of 36 males and 64 females. In the student control group there were 61 males and 39 females while in the second control group 142 were male and 353 were female ( 5 people in the other control group did not indicate their gender).

Comparing the proportions of right-, mixed- and left handedness across the four groups: patients, family members, students and second controls:

Table 1 displays the cross-tabulation of the three groups: patients (patients and family members are paired and can't be used independently), student controls and other controls and their EHI results divided into 3 categories (Table 1).

The Pearson's chi square test statistic indicated a highly significant association between the patient group and student control group regarding handedness $\left(\chi^{2}=12.505\right.$, $p=0.002$ ) Highly significant associations were also found when the student control group was compared to the family members as well as the other control group. The reason for this significance is the unexpected high mixed handedness of the student control group.

The Pearson's chi square test statistic indicates that in this study there exists a highly significant association between group and handedness $\left(\chi^{2}=31.174, p<0.0001\right)$ when comparing the patients, family members and student control group. When comparing the patients, family members and second control group; the Pearson's chi square test statistic indicates that no significant difference in terms of handedness was found $\left(\chi^{2}=3.935, p=0.416\right)$.

Comparing the proportions of right- and non-right handedness across the four groups: patients, family members, students and second controls:

Table 2 displays the cross-tabulation of the three groups: patients (patients and family members are paired and can't be used independently), student controls and other controls; mixed- and left-handedness were combined into one category - namely, non-right handed (Table 2).

The patients and family members were dependent because of the patient-family pairing.

The McNemar test for paired data was used to test whether there was a difference in the proportions of family members and patients regarding non-handedness. It was found that there was no difference between the proportion of non-right handedness of family and patient members $(p=0.248)$

Exactly the same pattern in results was found when analyzing the groups with two categories namely rightand non-right handedness versus three categories namely right-handed, mixed-handed and left-handed. When the student control group was compared with any other group a highly significant association existed.

\section{Gender and handedness}

The relationship between gender and handedness of the patient group:

The association between handedness and gender when only considering right- versus non-right handedness was investigated. There was a statistically significant association between gender and handedness at the 5\% level of significance (Fisher's Exact test 2-sided, $p=0.039$ ). The reason for the significant association is that among the patients more females (9) than the expected 5.1 and fewer males (8) than the expected 11.9 under the null hypothesis of independence were non-right handed.

The relationship between gender and right-, mixed- and left handedness across the patients and student control group:

A highly significant association was found by using Pearson's chi square test statistic between the male gender of the patients and student control group regarding handedness $\left(\chi^{2}=10.191, p=0.006\right)$. The same effect was observed for the females, but the test was not significant due to the small sample size.

The relationship between gender and right-, mixed- and left handedness across the patients and other control group:

No significant association was found by using Pearson's chi square test statistic between the male gender of the patients and the other control group regarding handedness. A significant association was found by using Fisher's exact test statistic between the female gender of the patients and other control groups regarding handedness $(p=0.01)$. If the mixed- and left-handed females were combined into one category - namely, non-right handed there was a highly significant association between the female gender patients and the other control group regarding handedness $(p=0.06)$.

\begin{tabular}{|c|c|c|c|c|c|c|}
\hline & & & \multicolumn{3}{|c|}{ Groups } & \multirow[t]{2}{*}{ Total } \\
\hline & & & Patients & Control- Students & Control - Other & \\
\hline \multirow{9}{*}{ EHI result } & \multirow{3}{*}{ Right-handed } & Count & 82 & 64 & 425 & 571 \\
\hline & & Row \% & $14.4 \%$ & $11.2 \%$ & $74.4 \%$ & $100.0 \%$ \\
\hline & & Column $\%$ & $82.0 \%$ & $64.0 \%$ & $85.0 \%$ & $81.6 \%$ \\
\hline & \multirow{3}{*}{ Mixed-handed } & Count & 10 & 30 & 46 & 86 \\
\hline & & Row \% & $11.6 \%$ & $34.9 \%$ & $53.5 \%$ & $100.0 \%$ \\
\hline & & Column \% & $10.0 \%$ & $30.0 \%$ & $9.2 \%$ & $12.3 \%$ \\
\hline & \multirow{3}{*}{ Left-handed } & Count & 8 & 6 & 29 & 43 \\
\hline & & Row \% & $18.6 \%$ & $14.0 \%$ & $67.4 \%$ & $100.0 \%$ \\
\hline & & Column \% & $8.0 \%$ & $6.0 \%$ & $5.8 \%$ & $6.1 \%$ \\
\hline \multirow{3}{*}{\multicolumn{2}{|c|}{ Total }} & Count & 100 & 100 & 500 & 700 \\
\hline & & Row \% & $14.3 \%$ & $14.3 \%$ & $71.4 \%$ & $100.0 \%$ \\
\hline & & Column $\%$ & $100.0 \%$ & $100.0 \%$ & $100.0 \%$ & $100.0 \%$ \\
\hline
\end{tabular}




\begin{tabular}{|c|c|c|c|c|c|c|}
\hline & & & & Groups & & Total \\
\hline & & & Patients & Control-Students & Control - Other & \\
\hline \multirow{6}{*}{ EHI result } & \multirow{3}{*}{ Right-handed } & Count & 82 & 64 & 425 & 571 \\
\hline & & Row \% & $14.4 \%$ & $11.2 \%$ & $74.4 \%$ & $100.0 \%$ \\
\hline & & Column \% & $82.0 \%$ & $64.0 \%$ & $85.0 \%$ & $81.6 \%$ \\
\hline & \multirow{3}{*}{ Non-Right-handed } & Count & 18 & 36 & 75 & 129 \\
\hline & & Row \% & $14.0 \%$ & $27.9 \%$ & $58.1 \%$ & $100.0 \%$ \\
\hline & & Column \% & $18.0 \%$ & $36.0 \%$ & $15.0 \%$ & $18.4 \%$ \\
\hline \multirow{3}{*}{\multicolumn{2}{|c|}{ Total }} & Count & 100 & 100 & 500 & 700 \\
\hline & & Row \% & $14.3 \%$ & $14.3 \%$ & $71.4 \%$ & $100.0 \%$ \\
\hline & & Column \% & $100.0 \%$ & $100.0 \%$ & $100.0 \%$ & $100.0 \%$ \\
\hline
\end{tabular}

The age at onset of illness and handedness:

The average age at onset of the illness for males in this study group was 22.9 years (standard deviation 5.9 years) with a median of 21 years. For the female patients the average age at onset of the illness was 23.9 years (standard deviation 9.2 years) with a median of 22 years. Age at onset of illness was categorized into two groups: those whose illness started at an age younger than 18 and those whose illness started at age 18 and older. No statistically significant difference between age at onset of illness and handedness could be established $(\mathrm{t}=-0.666, \mathrm{p}=0.507)$.

Statistical analysis with the EHI score in its raw format continuous scale:

The EHI scores for the 3 independent groups: patients, students control and other control; as well as the family members as calculated in the questionnaire were used. Consider the percentage histograms for the 4 groups in figure 1: patients (100), family members (100), student control group (100) and other control group (500) (Figure 1).

It is clear from the percentage histograms that the handedness pattern of the student control group differed from the other three groups. The Kruskal-Wallis Test was done to test if the median scores between the 3 independent groups (family members and patients were paired) were equal. The distribution of EHI scores for each group was extremely skew to the left (majority were right handed) thus not symmetrical - therefore a one way ANOVA could not be used. Since $p<0.001$, there existed enough evidence to conclude that there was a difference among the patient and two control groups based on the EHI scores. The Mann Whitney U test was used to do pair wise comparisons of the median raw scores across the Patient group versus the two control groups as well as between the two control groups. A highly significant difference $(p<0.0001)$ was found between the median EHI scores of the patient group and the student control group. A highly significant difference $(p<0.0001)$ was found between the median EHI scores of the student control group and the other control group. No significant difference existed between the median EHI scores of the patient group and the other control group. These results perfectly supported the results founded with the cut-off points.

The Wilcoxon Signed ranks test revealed a significant difference $(p=0.042)$ between the median EHI scores of the patients and the family members. It can also be concluded that the median EHI scores of the patients were significantly lower than their paired family members $(p=0.021)$. At first glance this result may seem to be in contrast with the result of the McNemar test where categories based on the cut-off points were compared. This contrast can be explained by noting that the significance of McNemar's test is dependent on the specific cut-off points chosen.

\section{Discussion}

The present study tested the hypothesis that patients with schizophrenia/schizoaffective disorder are more non-right handed (mixed handed or left handed) than their firstdegree relatives and two control groups. Contrary to this hypothesis, in the present study, patients suffering from schizophrenia/schizoaffective disorder taken as a whole showed less non-right handedness compared to the student control group but no significant difference in handedness compared to their non-affected relatives and the second control group when using the EHI cut- off point of $+/-60$. When comparing the raw EHI scores of the patients and the family members, the median EHI scores of the patients were significantly lower than their paired family members; meaning that the patients were weaker right-handed. Interestingly and in contrast to this finding, the student control group showed a significant non-right handedness compared to the patient and family groups. In the literature, the majority of studies investigating the handedness of patients with schizophrenia showed a leftward shift in the handedness distribution compared to the controls $8,9,23,24$. Our results are incompatible with a large number of these previous studies that reported a leftward shift in the handedness distribution of patients with schizophrenia compared to controls.

The finding that the medical student control group was more non-right handed than the other two groups was an interesting and unexpected finding. Possible explanations for this finding are discussed. The medical students selected for the MBChB course are top achievers at school level, where creative thinking is of prime importance. In a previous study on handedness and gender differences in intelligence, evidence was collected from the Medical College Admission Test (MCAT) that revealed two intriguing results that require additional research and confirmation: first, there was a preponderance of left handers among those who attained the highest percentage scores on the verbal reasoning subtest of the MCAT - a fact that led to a disproportionately high percentage of left handers admitted to medical school; second, the abovementioned first result was modified by the finding that a higher percentage of right handers were found among the highestscoring group on the writing sample ${ }^{24}$. These results suggest that for the most intellectually gifted individuals, 
Figure 1: Percentage histograms comparing the handedness across the four groups with the EHI score in its raw format on a continuous scale

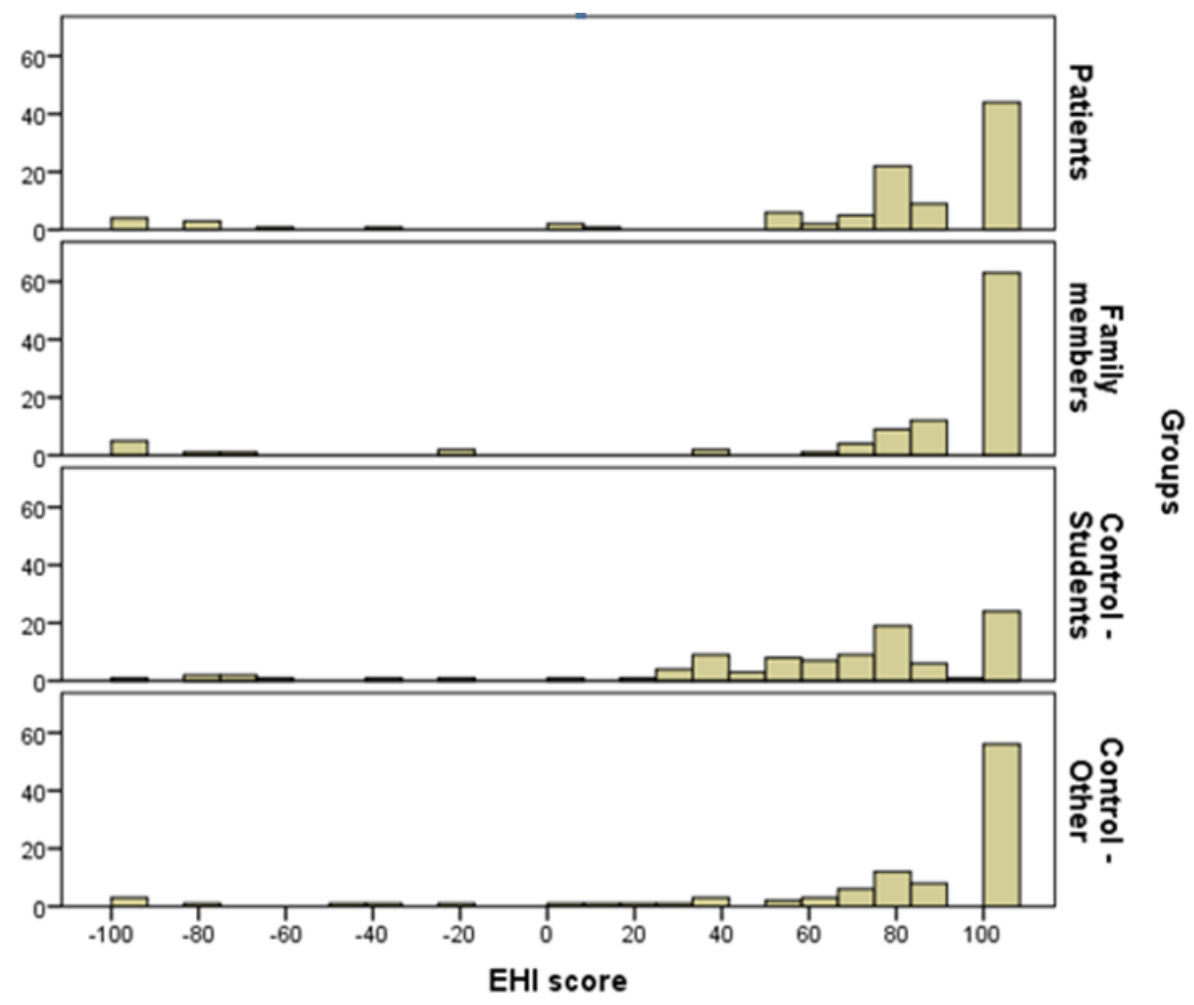

different patterns of cerebral organization are optimal for different types of cognitive tasks. The understanding of the way gender and lateral preference reflect brain and other neural processes is still incomplete. Converging evidence across studies of gender differences, laterality, cerebral activity and morphology, and cognitive abilities suggests that structural and organizational differences in the brains of males and females and right- and left handers are systematically related to different patterns of cognitive abilities. The largest differences occur in the extreme tails of the distributions; i.e. retarded and intellectually gifted portions of the intelligence range ${ }^{24}$.

Furthermore, in a study by Antonarakis that determined whether the orientation of stethoscopes when placed around the neck by physicians is a random occurrence or if it represents a lateral preference, it was found that the preference correlated with handedness, footedness, earedness and hand clasping ${ }^{25}$. Thirty-five per cent (35\%) of participants demonstrated left-stethoscope-orientation type. In a meta-analytic review of lateralization of creativity, the results support the notion of right-hemispheric superiority in creative thinking. The right hemispheric specialization was found to be closely associated with different thinking processes necessary in the context of creative problem solving; e.g. requirement of a global thinking style ${ }^{26}$. These results speak strongly in favour of right-hemisphere involvement in the process of abstract thinking.

Interestingly, our study found that there was a significant association between female gender and non-right handedness in the patient group but no gender difference was found in the family- and student control groups. This finding is in contrast with those of some studies that have found no gender differences in relation to handedness in schizophrenia whereas several authors have found an association between non-right handedness and the male gender ${ }^{11-15}$. Our study found no association between age at onset of illness and handedness in schizophrenia/ schizoaffective disorder. This finding is inconsistent with the view that lateralization is anomalous in schizophrenia early in the course of illness, as was demonstrated in the study by Collinson et al. ${ }^{18}$.

It has been proposed that schizophrenia is associated with a reduction or reversal of cerebral dominance (as measured by handedness) on the basis of functional and anatomical evidence. Francks et al. claim to have identified "The first potential genetic influence on human handedness, namely the LRRTM1, which showed to be over transmitted paternally to patients with schizophrenia/schizoaffective disorder... and the first putative genetic effect on variability in human brain asymmetry" and a gene "that underlies much of human cognition, behaviour and emotion" 19 .

\section{Study Limitations}

First, the assessment and measurement of handedness in relation to schizophrenia has varied across studies, limiting the comparability of results. Presumably, the discrepancy found in the distribution of handedness in patients with schizophrenia/schizoaffective disorder may be due to different methods used in handedness assessment and classification. The EHI is, however, the most widely used questionnaire and is widely accepted as the standard in eliciting handedness data ${ }^{27}$ and was, therefore, used in our study. The EHI could, however, have limited the comparability of our study results to those of other studies that used a different scale or different cut-off LQ scores to classify handedness. The prevalence of handedness changes substantially depending on the definition used. In a study by Dragovic et al. the researchers used a cutoff point that best separated mixed handedness from 
single left- and right handedness ${ }^{23}$. The best cut-off point was found to be between $0+/-50$ to $0+/-70$ with the best statistical agreement at the $0+/-60$ cut-off point. For this reason, the $0+/-60$ cut-off, which could have narrowed the mixed-handedness criteria, was used in our study.

To address this limitation, the Mann Whitney U test was used to do pair wise comparisons of the median raw scores across the patient group versus the two control groups and family members. These results supported the results founded with the cut-off points. However it was interesting to find that with the Wilcoxon Signed ranks test, the median EHI scores of the patients were significantly lower than their paired family members. At first glance this result may seem to be in contrast with the result of the McNemar test where categories based on the cut-off points were compared. This contrast can be explained by noting that the significance of McNemar's test is dependent on the specific cut-off points chosen.

Second, the manner in which the responses were elicited (verbal versus demonstration) could also have affected the classification of handedness. The findings in the literature on which method is the more reliable and valid are inconsistent. In the Edinburgh High Risk for Schizophrenia Study, an evaluation of handedness using two different scales, the Annet Scale and the Edinburgh Handedness Inventory were performed ${ }^{28,29}$. The evaluation required using verbal report and demonstration to elicit responses. It was not clear if any one of the scales was more valid and reliable than any other and verbal report of hand preference elicited less 'right' responses and more 'mixed' responses than demonstration trials $^{30}$. However, from the results reported in the literature, it appears that the most reliable method of eliciting lateral preference is by demonstration of the action rather than by verbal report ${ }^{31}$. Unfortunately, for the sake of convenience for the researchers and study participants, a significant portion of the data collection and handedness assessments were done telephonically - i.e. verbally - in our study. These telephonic assessments of handedness may have affected the reliability of the results because of the inherent subjectivity of the handedness tasks and also because we relied on the participants' interpretation of the question, as well as their ability to imagine themselves performing the particular task.

Crow et al. have raised a number of concerns with regard to the LRRTMl study undertaken by Francks et al. ${ }^{19,32}$ Crow et al. believe that the search for the right-shift-factor and cerebral dominance gene is important and relevant to both schizophrenia and other psychoses and dyslexia, but were unconvinced by the case that Francks et al. have presented for LRRTMl. Crow et al. in fact believe that the cerebral dominance gene is on the $\mathrm{X}$ and $\mathrm{Y}$ chromosome, specifically the gene pair Protocadherin X and Protocadherin Y, which was created in the course of hominid evolution ${ }^{32}$.

Crow et al. summarized the main errors in the LRRTMl study as follows: The assumption of heterogeneity without independent justification; inappropriate application of a technique designed to detect rare sequence variants to a problem relating to the population as a whole; neglect of what is already known about the anatomical and physiological correlates of the disorder in question; and failure to recognize that psychosis relates to the characteristic that defines Homo Sapiens as a species and therefore must have been subject to significant change in the course of hominid evolution ${ }^{32}$.
In response to the critique published, there was a personal response by Francks in which he argued that Crow et al. have re-stated a long-standing, pseudo-autosomal, singlegene hypothesis for handedness, schizophrenia, and brain asymmetry $^{2,32}$. Francks argues that single-gene theories have not found broad support among the psychiatricgenetics community, as most researchers consider such models unrealistic for complex traits such as schizophrenia and insufficient to explain genetic epidemiological, linkage and association data and data on environmental influences ${ }^{2}$.

A question that flows from the abovementioned arguments and controversies is: "What is the implication of our study for the LRRTMl?" study by Francks et al. ${ }^{19}$.

Further limitations include the fact that the patient-firstdegree-relative pairs were not statistically randomized before they were contacted. The DIGS summary report has over 600 patients of the Afrikaner Founder population diagnosed with schizophrenia, but tracing the patients and/or their first-degree-relatives proved to be very difficult. The authors had to resort to using the first 100 patient-relative pairs that were available. Although they were not randomized, their handedness was not known to the researchers before inclusion in the study. It could also be argued that the use of a non-randomized/convenient sample group was a confounding factor.

The use of medical students as a control group could be a further limitation as it may have caused selection bias, as most of the medical students are intellectually gifted and may have different patterns of cerebral organization that are needed for optimal performance of different types of cognitive tasks. In light of this, a larger more suitable control group has been selected, taking into account the economic background and/or level of education of the participants.

\section{Conclusion and Recommendations}

Even though the majority of studies investigating the handedness of patients with schizophrenia showed a leftward shift in the handedness distribution compared to controls, non-right handedness was not clinically established in this study. Interestingly, an association between non-right handedness and female gender in the patient group was established. Whether or not LRRTMl on chromosome 2pl2 is indeed the genetic basis of handedness remains unclear.

It is the authors' recommendation that researchers should adopt a standard definition of handedness that would allow for comparability of results across studies.

\section{Acknowledgements}

The authors would like to extend their gratitude to the patients, family members and controls who participated in this study. The study was partially funded by a NRF Incentive Funding grant to J.L.R.

References

1. McManus C, Nicholas M, Vallortigara G. Is LRRTM 1 the gene for handedness? Terality 2009; 14: 1-2.

2. Francks C. Understanding the genetics of behavioural and psychiatric traits will only be achieved through a realistic assessment of their complexity. Laterality 2009; 14: 11-16.

3. 3. Kenneth KGD, Cannon M, Gilvarry CM, Jones PB, Murray RM. Schizophrenic patients and their first-degree relatives show an excess of mixed handedness. Schiz Res 1999; 39: 167-176. 
4. Delisi LE, Svetina C,Raz K, Shields G,Wellman N, et al. Hand preference and hand skill in families with schizophrenia. Laterality 2002; 7: 32 1-332.

5. Dollfus S, Alary M, Razafimandimby A, Prelipceanu $D$, Rybakowski JK, et al. Familial sinistrality and handedness in patients with first episode schizophrenia: the EUFEST study. Laterality 2012; 17:217-24.

6. Dragovic M, Hammond G. Handedness in schizophrenia: a quantitative review of evidence. Acta Psychiatrica Scand 1999; 111:410-419.

7. Satz P, Green MF. Atypical handedness in schizophrenia: some methodological and theoretical issues. Schiz Bull 1999; 25: 63-78.

8. Sommer I, Aleman A, Ramsey $N$, Bouma $A$, Khan $R$. Handedness, language lateralization and anatomical asymmetry in schizophrenia. Br J Psychiatry 2001; 178: 344-351.

9. Preti A, Sardu C, Piga A. Mixed-handedness is associated with the reporting of psychotic-like beliefs in a nonclinical Italian sample. Schiz Res 2007; 92: 15-23.

10. Sadock BJ, Sadock VA. Synopsis of psychiatry, (10thed.) Lippincott Williams \& Wilkins, Philadelphia, USA, 2007.

11. Buijsrogge JJA, Benali K, Brazo P, Dollfus S. Sinistrality in schizophrenia. Schiz Res 2002; 55:303-306.

12. Schiffman J, Pestle S, Mednick S, Ekstrom M, Sorensen $H$, et al. Childhood laterality and adult schizophrenia spectrum disorders: a prospective investigation. Schiz Res 2005; 72: 151-560.

13. Manoach DS. Handedness is related to formal thought disorder and language dysfunction in schizophrenia. $J$ Clin Exp Neuropsychol 1994; 16: 2-14.

14. Sperling $W$, Martus $P$, Barocka A. Non-right-handedness and obstetrical complications in paranoid hallucinatory schizophrenics. Psychopathol 1999; 32: 267-276.

15. O'Callaghan E, Buckley P, Madigan C, Redmond O, Stack $J P$, et al. The relationship of minor physical anomalies and other putative indices of developmental disturbance in schizophrenia to abnormalities of cerebral structure on magnetic resonance imaging. Biol Psychiatry 1995; 38: 516-524.

16. Lishman WA, Mc Meekan ER. Hand preference patterns in psychiatric patients. Br J Psychiatry 1976; 129: 158-166.

17. Oddy HC, Lobstein TJ. Hand and eye dominance in schizophrenia. Br J Psychiatry 1972; 120: 331-332.

18. Collinson SL, Phillips TJ, James ACD, Quested DJ, Crow TJ. Is lateral bias anomalous in early-onset schizophrenia? Selected comparisons with normal populations. Psychiatry Res 2004; 25: 219-224.
19. Francks C, Maegawa S, Lauren J, Abrahams BS, VelayosBaeza $A$, et al. LRRTMl on chromosome $2 p 12$ is a maternally suppressed gene that is associated paternally with handedness and schizophrenia. Mol Psychiatry 2007; 12: 1129-1139.

20. Nurnberger JI, Blehar MC, Kaufmann CA, York-Cooler C, Simpson SG, et al. Diagnostic interview for genetic studies, rationale, unique features, and training. Arch Gen Psychiatry 1994; 51: 949.

21. Karayiorgou M, Torrington M, Abecasis GR, Pretorius $H$, Robertson $B$, et al. Phenotypic characterization and genealogical tracing in an Afrikaner schizophrenia database. Am J Med Gen 2004; 124B: 20-28.

22. Dragovic M. Categorization and validation of handedness using latent class analysis. Acta Neuropsychiatrica 2004; 16: 212-218.

23. Dragovic $M$, Hammond G. Laterality phenotypes in patients with schizophrenia, their siblings and controls: associations with clinical and cognitive variables. $\mathrm{Br} J$ Psychiatry 2005; 187: 221-228.

24. Halpern DF, Haviland MG, Killian CD. Handedness and sex differences in intelligence: evidence from the Medical College Admission Test. Brain Cogn 1998; 38: 87-101.

25. Antonarakis ES. Orientation of the stethoscope around the neck: a random phenomenon or an indicator of cerebral lateralization? Cross-sectional survey. Laterality 2006; 11 : 287-293.

26. Mihov KM, Denzler M, Foster J. Hemisphere specialization and creative thinking: a meta-analytic review off lateralization of creativity. Brain Cogn 2010; 72: 442-448.

27. Ransil BJ, Schachter SC. Test-retest reliability of the Edinburgh Handedness Inventory and global handedness preference measurement, and their correlation. Percept Motor Skills 1994; 79: 1355-1372.

28. Annett M. A classification of handpreference by association analysis. Br J Psychol 1970; 61: 303-321.

29. Oldfield RC. The assessment and analysis of handedness: the Edinburgh inventory. Neuropsychol 1971; 9: 97-113.

30. Byrne $M$, Clafferty RA, Cosway $R$, Grant E, Hodges $A$, et al. Measurement of lateral preference and schizophrenia: results of the Edinburgh High-Risk Study and methodological issues. Psychiatry Research 2004; 125: 205-217.

31. Beaton AL. Left side, right side. New Haven: Yale University Press, USA, 1985.

32. Crow TJ, Close JP, Daggnall AM, Priddle TH. Where and what is the right shift factor or cerebral dominance gene? A critique of Francks et al. 2007. Laterality 2009; 14: 3-10. 